\title{
Free-Standing Mechanical and Photonic Nanostructures in Single-Crystal Diamond
}

\section{Citation}

Burek, Michael J., Nathalie P. de Leon, Brendan J. Shields, Birgit J. M. Hausmann, Yiwen Chu, Qimin Quan, Alexander S. Zibrov, Hongkun Park, Mikhail D. Lukin, and Marko Lon?ar. 2012. "Free-Standing Mechanical and Photonic Nanostructures in Single-Crystal Diamond." Nano Letters 12 (12) (December 12): 6084-6089. doi:10.1021/nl302541e.

\section{Published Version}

doi:10.1021/nl302541e

\section{Permanent link}

http://nrs.harvard.edu/urn-3:HUL.InstRepos:33717988

\section{Terms of Use}

This article was downloaded from Harvard University's DASH repository, and is made available under the terms and conditions applicable to Other Posted Material, as set forth at http:// nrs.harvard.edu/urn-3:HUL.InstRepos:dash.current.terms-of-use\#LAA

\section{Share Your Story}

The Harvard community has made this article openly available.

Please share how this access benefits you. Submit a story.

\section{Accessibility}




\title{
Free-Standing Mechanical and Photonic Nanostructures in Single- Crystal Diamond
}

\author{
Michael J. Burek, ${ }^{\dagger}$ Nathalie P. de Leon, ${ }^{\ddagger}, \S$ Brendan J. Shields, ${ }^{\ddagger}$ Birgit J. M. Hausmann, ${ }^{\dagger}$ Yiwen Chu, \\ Qimin Quan, ${ }^{\dagger}$ Alexander S. Zibrov, ${ }^{\dagger}$ Hongkun Park, ${ }^{\dagger, \S}$ Mikhail D. Lukin, ${ }^{\ddagger}$ and Marko Lončar*, ${ }^{\dagger}$ \\ ${ }^{\dagger}$ School of Engineering and Applied Sciences, Harvard University, 29 Oxford Street, Cambridge, Massachusetts 02138, United States \\ ${ }^{\ddagger}$ Department of Physics, Harvard University, 17 Oxford Street, Cambridge, Massachusetts 02138, United States \\ ${ }^{\S}$ Department of Chemistry and Chemical Biology, Harvard University, 12 Oxford Street, Cambridge, Massachusetts 02138, United \\ States
}

ABSTRACT: A variety of nanoscale photonic, mechanical, electronic, and optoelectronic devices require scalable thin film fabrication. Typically, the device layer is defined by thin film deposition on a substrate of a different material, and optical or electrical isolation is provided by the material properties of the substrate or by removal of the substrate. For a number of materials this planar approach is not feasible, and new fabrication techniques are required to realize complex nanoscale devices. Here, we report a three-dimensional fabrication technique based on anisotropic plasma etching at an oblique angle to the sample surface. As a proof of concept, this angled-etching methodology is used to fabricate free-standing nanoscale components in bulk single-crystal diamond, including nanobeam mechanical resonators, optical waveguides, and photonic crystal and microdisk cavities. Potential applications of the fabricated prototypes range from classical and quantum photonic devices to nanomechanical-based sensors and actuators.

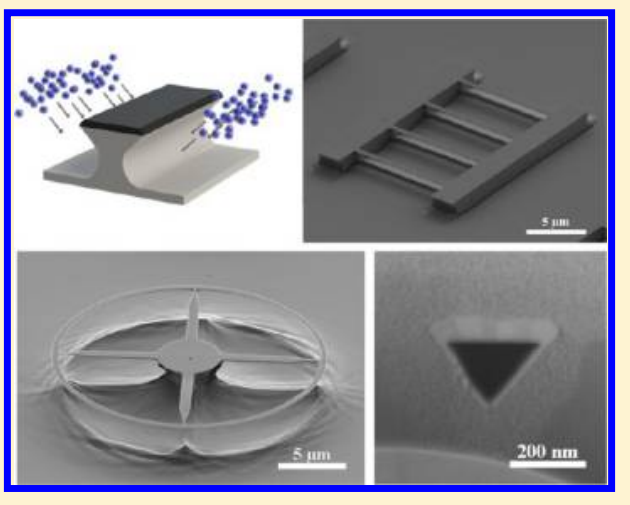

KEYWORDS: Nanofabrication, nanophotonics, nanomechanical systems, diamond, photonic crystal

$\mathrm{R}$ ealizing complex three-dimensional structures in a range of material systems is critical to a variety of emerging nanotechnologies. This is particularly true of nanophotonic and nanomechanical systems, both relying on free-standing small scale components. In the case of nanomechanics, necessary mechanical degrees of freedom require physically isolated structures, such as suspended beams, cantilevers, and membranes. ${ }^{1}$ For nanophotonics, elements like waveguides and photonic crystal cavities rely on light confinement provided by distributed Bragg reflection or total internal reflection, which requires refractive index contrast between the device and the surrounding medium (often air). ${ }^{2,3}$ Such suspended nanostructures are typically fabricated in a heterolayer structure, comprising of device (top) and sacrificial (middle) layers supported by a substrate (bottom), using standard surface nanomachining techniques. ${ }^{4,5}$ A selective, isotropic etch is then used to remove the sacrificial layer, resulting in free-standing devices. While high-quality, crystalline, thin film heterolayer structures are readily available for silicon (as silicon-oninsulator (SOI)) or III-V semiconductors (i.e., GaAs/AlGaAs), there remains an extensive list of materials with attractive electro-optic, piezoelectric, quantum optical, and other properties for which high quality single-crystal thin film heterolayer structures are not available. These include complex metal oxides like lithium niobate $\left(\mathrm{LiNbO}_{3}\right)$, silicon-based compounds such as silicon carbide ( $\mathrm{SiC}), \mathrm{III}-\mathrm{V}$ nitrides including gallium nitride $(\mathrm{GaN})$, and inert single-crystals such as diamond.
Diamond is especially attractive for a variety of nanoscale devices due to its exceptional physical and chemical properties, including high mechanical hardness, Young's modulus, and thermal conductivity. ${ }^{6}$ Optically, it is transparent over a wide wavelength range (from $220 \mathrm{~nm}$ to the far-infrared), has a high refractive index $(n \sim 2.4)$ and large Raman gain, ${ }^{6}$ and is host to a vast inventory of luminescent defect centers. ${ }^{7}$ Specifically, the negatively charged nitrogen-vacancy defect in diamond has recently gained significant attention due to its operation as a stable source of single photons at room temperature ${ }^{8,9}$ and as an optically addressable solid-state spin-qubit. ${ }^{10-12}$ Diamond has many potential applications ranging from radio frequency NEMS ${ }^{13,14}$ to all-optical signal processing and quantum optics. ${ }^{11,12}$ However, lack of scalable nanofabrication techniques capable of realizing complex three-dimensional nanostructures in diamond has been the major limiting factor to more widespread application of diamond in nanoscale science and technology. While focused ion beam (FIB) milling ${ }^{15-20}$ and crystal ion-slicing ${ }^{13,14,20-29}$ have been used to realize nanoscale mechanical or optical structures in single-crystal diamond, both techniques generally compromise the final material quality due to fabrication-induced imperfections, which often limits device

Received: July 10, 2012

Revised: November 13, 2012

Published: November 19, 2012 
(a)

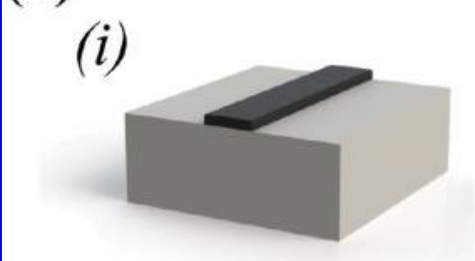

(b)

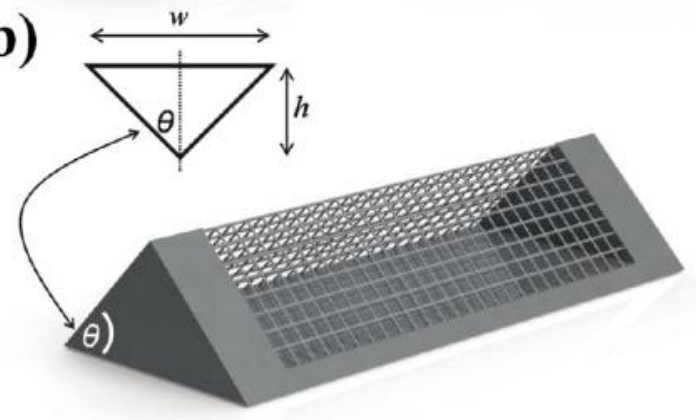

(ii)

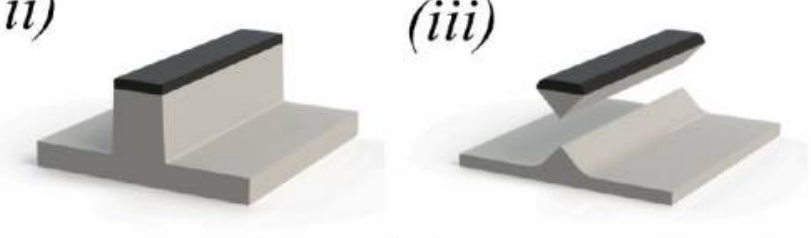

(c) (iv)

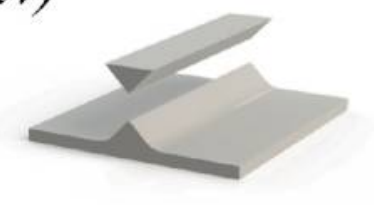

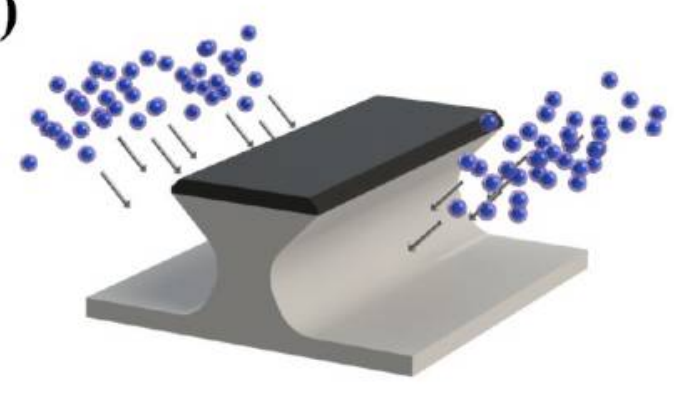

Figure 1. (a) Angled-etching fabrication schematic: (i) an etch mask was defined by standard electron beam lithography and thin film deposition techniques, (ii) the etch mask pattern was then transferred into diamond substrate by conventional top down plasma etching, (iii) angled-etching is then employed to realize suspended nanobeam structures, (iv) residual etch mask is removed. (b) Schematic of triangular prism Faraday cage design with inset showing the relationship between the prescribed etch angle and the nanobeam bottom apex. (c) Illustration of angled-etching from two directions accomplished with the triangular prism Faraday cage design.

performance. Recently, significant progress toward a diamondon-insulator platform has been made via thinning bulk singlecrystal diamond slabs (typically 5-20 $\mu$ m thick) adhered to a supporting wafer. ${ }^{19,30-33}$ Though this approach is quite promising, the difficulty in obtaining uniform, large area single-crystal diamond films limits its scalability. As such, continued investigation into new fabrication methods for freestanding nanostructures in bulk single-crystal diamond is necessary.

In this work, a novel bulk nanomachining approach for realizing suspended nanoscale mechanical and photonic elements is presented, with single-crystal diamond used as the example material system. Established planar fabrication processes, including electron beam lithography and anisotropic plasma etching, are employed, but in such a way as to produce suspended triangular cross-section nanobeam structures directly from single-crystal bulk diamond substrates. Figure 1a illustrates our approach to realize suspended nanobeam structures. Previously, free-standing triangular cross-section nanobeam structures have been proposed ${ }^{34}$ and demonstrated $^{18}$ in single-crystal diamond via FIB milling. The fabrication methodology presented here is a natural extension of this work, but with FIB-induced artifacts eliminated. These include $\mathrm{Ga}^{+}$ implantation and crystal damage, redeposited material, tapered sidewalls, one-of-a-kind nature of fabricated devices, and an inherently slow fabrication process.

Single-crystal diamond substrates (Element Six) were first cleaned in a boiling mixture consisting of equal parts sulfuric acid, nitric acid, and perchloric acid. Following the acid clean, a $\sim 200 \mathrm{~nm}$ thick titanium etch mask was defined on the prepared diamond substrate using electron beam evaporation and electron beam lithography techniques. Oxygen-based plasma etching was then carried out in a two-part process. First, a conventional top down anisotropic plasma etch was used to transfer the etch mask pattern into the bulk diamond. ${ }^{35}$ The diamond substrate was etched to a depth near $600 \mathrm{~nm}$ in this step. Following this, a second anisotropic etch step was performed, but at an oblique angle to the substrate surface (referred hereafter as "angled-etching") to release the nanostructures and yield the final suspended nanobeams. This angled-etching was achieved using a standard reactive ion etcher, by housing the sample inside a Faraday cage ${ }^{36,37}$ to shield it from electromagnetic fields. Although the Faraday cage has small grid openings on its surface, the effect of an external field is attenuated drastically within a small distance of the opening. Thus, the potential gradient in a plasma etching process builds up over the face of the Faraday cage and accelerates ions along a path perpendicular to the cage surface. Once the ions move past the metal grid and inside the cage, they are no longer accelerated and travel ballistically toward the substrate. Therefore, plasma ions may be directed to the sample surface at an oblique angle in multiple directions, determined by the geometry of the cage. ${ }^{37}$ One such Faraday cage design is a triangular prism structure shown schematically in Figure $1 \mathrm{~b}$. The triangular prism design allows for angled-etching in two simultaneous directions, as illustrated in Figure 1c. The incline angle of this Faraday cage $(\theta)$ defines the ion incidence angle relative to the surface normal (Figure $1 \mathrm{~b}$ inset). As such, the angle at the bottom apex of the final triangular cross-section nanobeams is $2 \theta$. Triangular prism Faraday cages with $\theta \sim 45^{\circ}$ were used in this work to fabricate suspended nanobeam mechanical and photonic structures. The bottom and triangular end plates of the Faraday cages were constructed out of aluminum, and the cage faces were aluminum mesh with 250 $\mu \mathrm{m}$ diameter mesh wire and a $2 \mathrm{~mm} \times 2 \mathrm{~mm}$ pitch. The Faraday cage height and length were $10 \mathrm{~mm}$ and $50 \mathrm{~mm}$, respectively, with the distance between cage bottom and the surface of the diamond substrate fixed at $\sim 3.5 \mathrm{~mm}$. While different configurations of Faraday cages can be used, it is important to emphasize that angled-etching may not be realized through simple tilting of the substrate within a plasma etcher without a Faraday cage. ${ }^{38,39}$ 


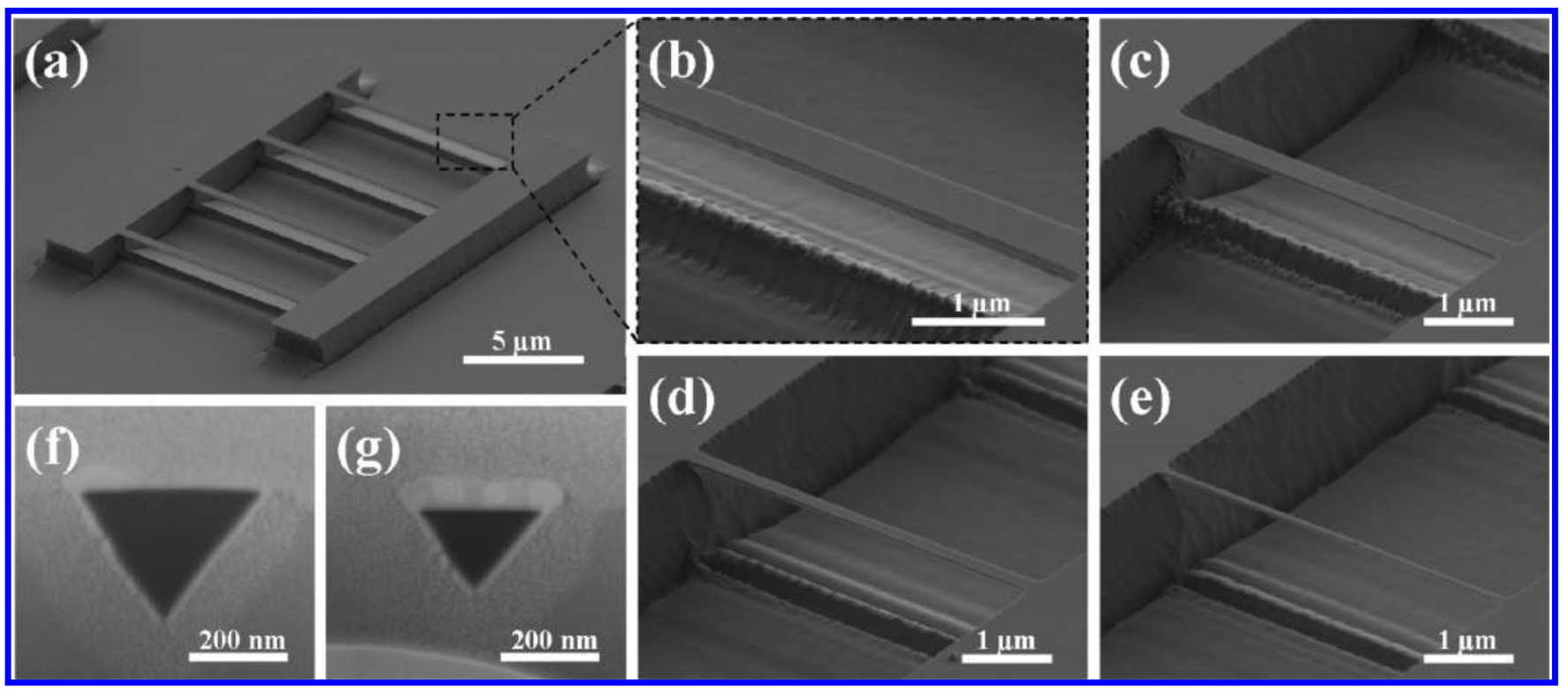

Figure 2. SEM images and an array of (a) suspended $\sim 500 \mathrm{~nm}$ wide solid diamond nanobeams fabricated with a triangular prism Faraday cage. Close-up SEM images of (b) $\sim 500 \mathrm{~nm}$, (c) $350 \mathrm{~nm}$, (d) $200 \mathrm{~nm}$, and (e) $75 \mathrm{~nm}$ wide solid diamond nanobeams. SEM images of FIB cross-sectioned (f) $\sim 350 \mathrm{~nm}$ and $(\mathrm{g}) \sim 250 \mathrm{~nm}$ wide solid diamond nanobeams. All SEM images were taken at a $60^{\circ}$ stage tilt.

Both the initial top-down etch and the angled-etch steps were performed in a UNAXIS Shuttleline inductively coupled plasma-reactive ion etcher (ICP-RIE) with the following parameters: $700 \mathrm{~W}$ ICP power, $100 \mathrm{~W}$ RF power, $50 \mathrm{sccm}$ $\mathrm{O}_{2}$ flow rate, $2 \mathrm{sccm} \mathrm{Cl}_{2}$ flow rate, and $10 \mathrm{mTorr}$ chamber pressure. Chlorine gas was included in the oxygen plasma diamond etch to prevent mask redeposition and subsequent roughening of the suspended feature. The trace amount of chlorine promotes the formation of volatile titanium chlorides, which eliminates micromasking due to ion bombardment of the titanium etch mask. However, it is important to note that the inclusion of chlorine may modify the diamond surface, which could ultimately influence the properties of nitrogen-vacancies or other color centers in close proximity to these surfaces. An approximate top-down etch rate of $200 \mathrm{~nm} / \mathrm{min}$ was measured under these plasma conditions, and a comparable rate was observed for angled-etching. Following the oxygen-based plasma etching, the remaining titanium etch mask was removed in concentrated hydrofluoric acid.

Figure 2 displays scanning electron microscopy (SEM) images of a range of suspended single-crystal diamond nanobeams fabricated by angled-etching with the triangular prism Faraday cage design. The nanobeam structures, which are examples of doubly clamped nanomechanical resonators or optical waveguides, are suspended above the diamond substrate with significant clearance $(\sim 2 \mu \mathrm{m})$. Fabricated suspended diamond nanobeams span up to $85 \mu \mathrm{m}$ long devices, with nanobeams widths ranging from $75 \mathrm{~nm}$ to over a micrometer wide. The nanobeams appear straight, in contrast to freestanding single-crystal diamond structures fabricated by crystal ion-slicing, ${ }^{13,14,27,29}$ which were observed to bow significantly due to residual fabrication-induced stress.

To characterize the etch angle of the fabricated structures, suspended nanobeams were cross-sectioned by FIB milling. Prior to ion milling, the suspended diamond structures were sputter coated with $\sim 150 \mathrm{~nm}$ of gold, which acted as an initial protection against FIB exposure. FIB cross sections began by coating a section of the free-standing nanobeams in a thick layer of platinum using ion beam-assisted deposition. A $30 \mathrm{kV}$ $\mathrm{Ga}^{+}$ion beam was then used to mill away the platinum and gold-coated diamond, exposing the triangular cross-section for SEM imaging.

Inspections of the FIB cross-sectioned diamond nanobeams, shown in Figure $2 \mathrm{f}$ and $\mathrm{g}$, revealed the expected triangular cross section. However, careful measurement of the apex half angle from a set of beams of various widths reveals an average etch angle of $\sim 35^{\circ}$. Consequently, the diamond nanobeams have a larger height/width ratio than expected from the $\theta \sim 45^{\circ}$ triangular prism Faraday cage. This discrepancy may be attributed to nonideal verticality resulting from the plasma etch parameters. A slight asymmetry is also observed in the triangular cross sections and was most likely due to a minor tilting of the substrate relative to the bottom of the Faraday cage. Since the ion trajectory is fixed by the mesh faces of the Faraday cage, by tilting the sample within the cage it is also possible to exploit this effect to adjust the cross-section of the final nanobeams. ${ }^{37}$

Beyond simple solid nanobeams, the angled-etching process presented here may also be used to fabricate nanobeam photonic crystal cavities (or simply "nanobeam cavities"), ${ }^{34}$ as shown in Figure 3. This nanobeam cavity architecture, ${ }^{40,41}$ based on a suspended nanobeam perforated with a chirped onedimensional lattice of air holes, features ultra high quality factors ( $Q$-factors) and ultrasmall mode volumes close to or below the diffraction limit $\left(V \sim(\lambda / n)^{3}\right)$. The prototype design in Figure 3, which is adapted from previous work, ${ }^{42,43}$ is based on a $400 \mathrm{~nm}$ wide nanobeam, $\theta \sim 35^{\circ}$ etch angle, lattice period of $220 \mathrm{~nm}$, and elliptical holes with a major radius decreased quadratically from $r_{1} \sim 100 \mathrm{~nm}$ at the center to $r_{30} \sim 60 \mathrm{~nm}$ at each end over 30 periods. Using finite-difference time-domain modeling, the nanobeam cavity design was confirmed to have a fundamental resonance at $\lambda \sim 637 \mathrm{~nm}$, with a $Q$-factor near $3 \times$ $10^{6}$ and mode volume of $2.26^{*}(\lambda / n)^{3}$. Interestingly, both the height and the width of angled-etched nanobeam cavities are determined by the width of the etch mask. This allows for truly three-dimensional scaling of the structure dimensions, and 


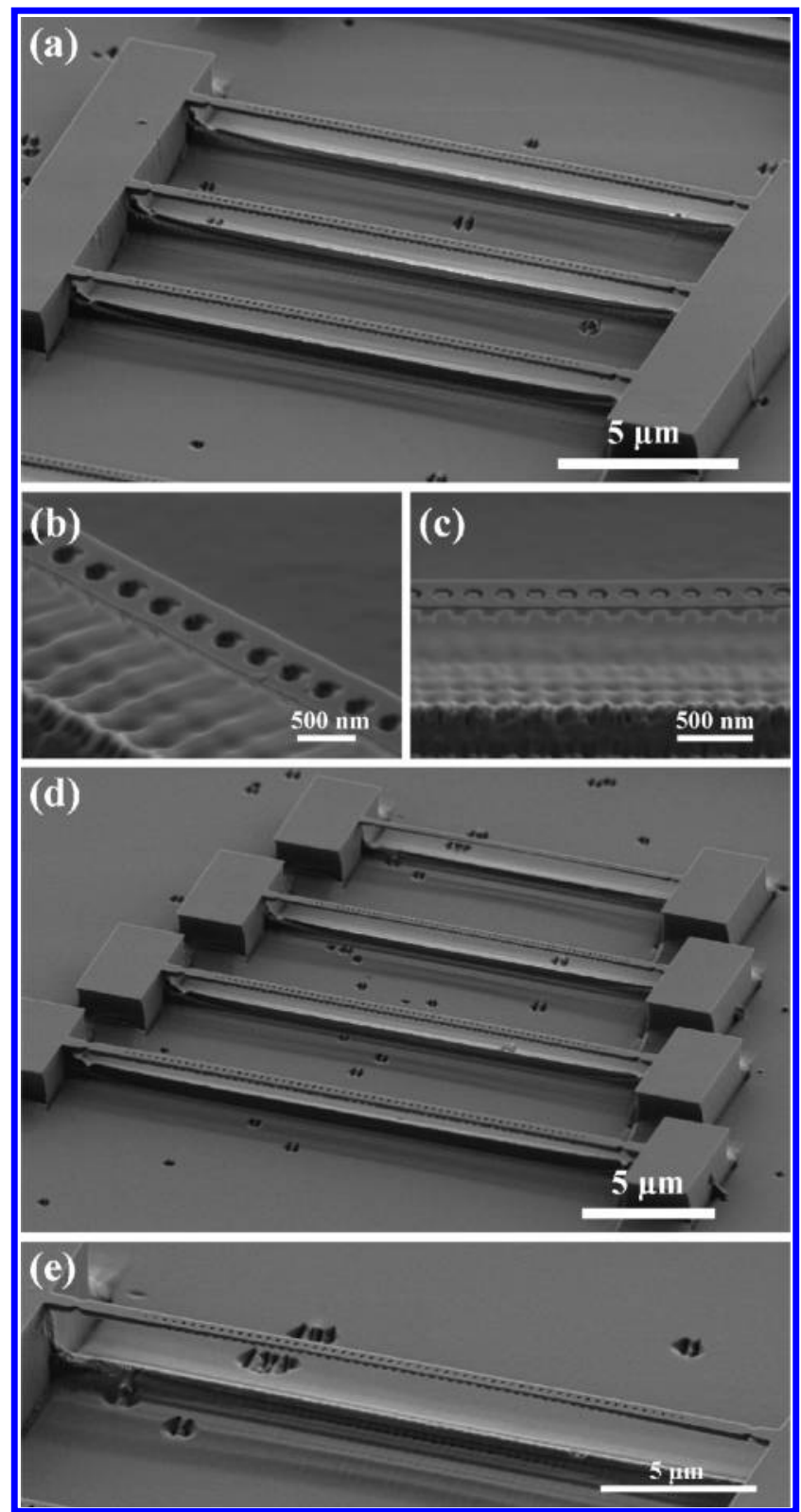

Figure 3. SEM images of (a) an array of fabricated diamond nanobeam cavity prototypes and $(\mathrm{b}-\mathrm{c})$ cavity region of an individual nanobeam cavity. (d) SEM image of an array of nanobeam cavities which are physically scaled from $75 \%$ to $135 \%$, and (e) zoomed in image of smallest, $75 \%$ scaled nanobeam cavity in the array. All SEM images were taken at a $60^{\circ}$ stage tilt.

therefore the tuning of the cavity resonance, simply by changing the dimensions of the etch mask. Similar flexibility is not available with photonic crystal cavities fabricated in thin films by surface nanomachining where the film thickness is a fixed parameter. To illustrate this point, Figure $3 \mathrm{e}$ shows an array of photonic crystal cavities with different physical scaling. Here, the prototype nanobeam cavity design dimensions are scaled from $75 \%$ to $135 \%$, in equal intervals. Figure $3 \mathrm{f}$ is a zoomed in image of the $75 \%$ scaled nanobeam cavity. Note that the etch pits observed in Figure 3 are the result of crystal defects in the diamond substrates. Such etch pits are not uncommon in standard grade diamond substrates. ${ }^{35}$

Other complex suspended nanobeam structures have also been realized with alternative Faraday cage designs. Figure $4 a$ is

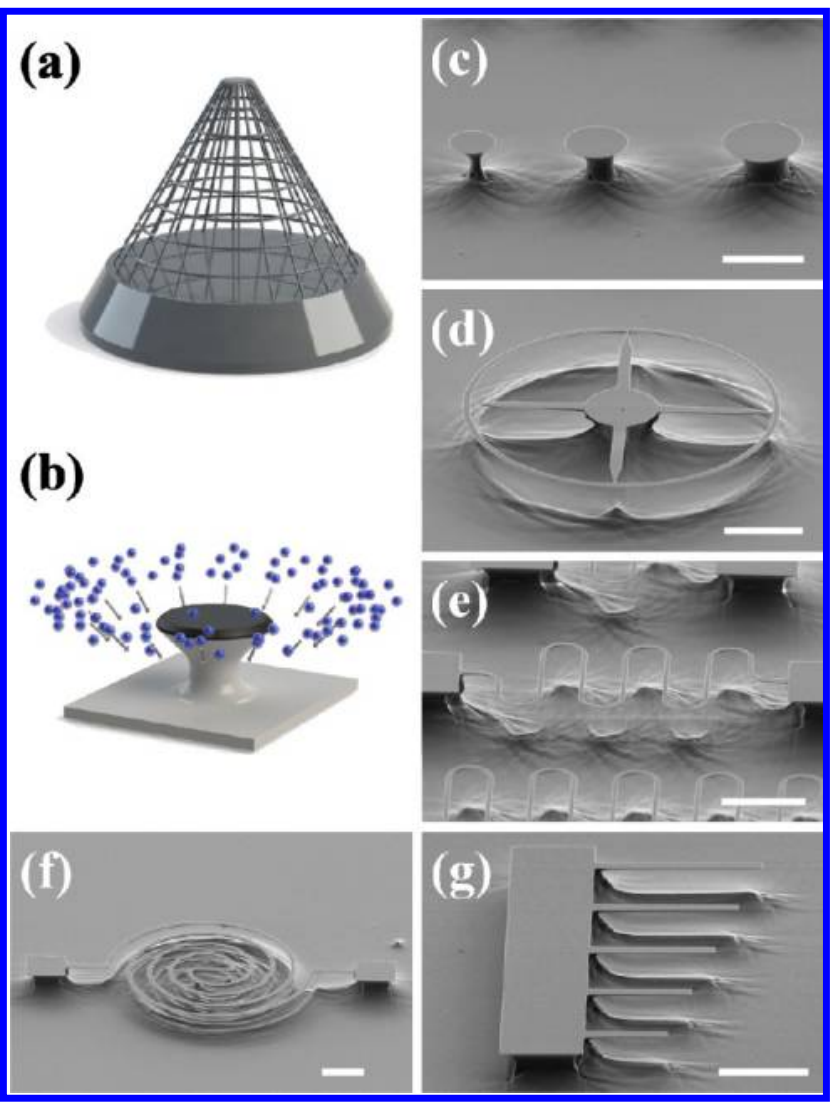

Figure 4. Schematic illustrations of (a) conical Faraday cage design and (b) angled-etching of the substrate from all directions. SEM images of (c) $\sim 3-5 \mu \mathrm{m}$ diameter undercut microdisks and (d) $\sim 500$ $\mathrm{nm}$ wide nanoring structure; (e) $\sim 500 \mathrm{~nm}$ wide curved and (f) $\sim 750$ $\mathrm{nm}$ wide spiral nanobeams; and $(\mathrm{g}) \sim 1 \mu \mathrm{m}$ wide nanobeam cantilevers. All SEM images were taken at a $60^{\circ}$ stage tilt. Scale bars correspond to $5 \mu \mathrm{m}$.

a schematic of a conical Faraday cage design, where angledetching occurs in all directions simultaneously, as illustrated in Figure $4 b$, which allows fabrication of suspended nanobeams oriented at arbitrary angles with respect to each other. Conical Faraday cages used in this work were constructed in a similar fashion as the triangular prism cages, again using a machined aluminum base and the identical aluminum mesh as before. The conical Faraday cage had a bottom diameter of $20 \mathrm{~mm}$ and an incline angle of $\sim 60^{\circ}$, with the diamond substrate surface fixed at a height of $\sim 10 \mathrm{~mm}$ from the cage bottom. Figure $4 \mathrm{c}$ and $\mathrm{d}$ show SEM images of micrometer-scale undercut diamond disks and suspended $500 \mathrm{~nm}$ wide nanorings. Angle-etched microdisks and suspended nanorings are expected to support whispering gallery modes, and as such, may operate as onchip optical resonators. ${ }^{30,32,44-46}$ These structures were fabricated using identical processing conditions as those for suspended nanobeams fabricated with the triangular prism Faraday cage. The noticeable asymmetry in the undercut microdisk features was likely the result of off-center placement of the substrate within the conical Faraday cage. FIB cross sections of free-standing nanorings (not shown here) revealed an etch angle of $\sim 50^{\circ}$, which is again $\sim 10^{\circ}$ off the prescribed etch angle, yielding a larger height/width ratio. To further demonstrate the versatility of angled-etching with a conical Faraday cage, curved and spiral suspended nanobeam structures were also fabricated, with representative images shown in 
Figure $4 \mathrm{e}$ and $\mathrm{f}$, respectively. It was also observed that angledetching with a conical Faraday cage was the preferred route to make cantilever structures, such as in Figure $4 \mathrm{~g}$.

In summary, a novel bulk nanomachining methodology for suspended nanobeam structures has been presented. The process is based on angled-etching achieved by housing a substrate within a Faraday cage during anisotropic plasma etching. Prototype devices fabricated in single-crystal diamond include suspended doubly clamped nanobeam mechanical resonators, optical waveguides, photonic crystal cavities, microdisks, and nanoring resonators. With the ability to create suspended nanobeam structures at virtually arbitrary angles and curvatures, the angled-etching process is a novel platform for realizing integrated photonic and mechanical structures in bulk media. This nanofabrication methodology will impact a wide variety of areas, ranging from classical and quantum photonic devices to NEMS-based sensors and actuators. Owing to the unique properties of diamond, and the current outstanding challenge to fabricate functional nanoscale devices in singlecrystal diamond, ${ }^{11}$ the presented fabrication procedure demonstrates a novel platform for integrated diamond-based nanomechanical and photonic devices. Furthermore, it allows for realization of novel all-diamond optomechanical devices ${ }^{47,48}$ that could take advantage of exceptional mechanical and optical properties of diamond. The current work is focused on characterization of fabricated nanoscale mechanical and photonic diamond prototype devices shown here, with experimental results to be presented in future dedicated manuscripts.

\section{AUTHOR INFORMATION}

\section{Corresponding Author}

*E-mail: loncar@seas.harvard.edu. Tel.: (617) 495-579. Fax: (617) 496-6404.

\section{Notes}

The authors declare no competing financial interest.

\section{ACKNOWLEDGMENTS}

This work was supported in part by the Defense Advanced Research Projects Agency (QuEST, QuASAR and QuINESS programs), the Hewlett-Packard Foundation, the NSF Center for Ultracold Atoms, as well as AFOSR MURI (Grant FA955009-1-0669- DOD35CAP). M.L. acknowledges support from the Sloan Foundation. Fabrication was performed at the Center for Nanoscale Systems (CNS) at Harvard University. M.J.B. is supported in part by the Natural Science and Engineering Council (NSERC) of Canada. N.P.d.L. is supported in part by the Element Six postdoctoral fellowship. B.J.M.H. gratefully acknowledges funding from the Harvard Quantum Optics Center (HQOC). The authors thank T.Y. Tsui for help during the initial phase of this project. M.J.B. thanks T. M. Babinec and $\mathrm{H}$. Atikian for useful discussions during preparation of the manuscript.

\section{REFERENCES}

(1) Ekinci, K. L.; Roukes, M. L. Rev. Sci. Instrum. 2005, 76 (6), 061101-12.

(2) Loncar, M.; Nedeljkovic, D.; Doll, T.; Vuckovic, J.; Scherer, A.; Pearsall, T. P. Appl. Phys. Lett. 2000, 77 (13), 1937-1939.

(3) Joannopoulos, J. D.; Johnson, S. G.; Winn, J. N.; Meade, R. D. Photonic Crystals: Molding the Flow of Light, 2nd ed.; Princeton University Press: Princeton, NJ, 2008.
(4) Carr, D. W.; Craighead, H. G. Pap. 41st Int. Conf. Electron, Ion, Photon Beam Technol. Nanofabrication 1997, 15, 2760-2763.

(5) Bogaerts, W.; Taillaert, D.; Luyssaert, B.; Dumon, P.; Campenhout, J. V.; Bienstman, P.; Thourhout, D. V.; Baets, R.; Wiaux, V.; Beckx, S. Opt. Express 2004, 12 (8), 1583-1591.

(6) Coe, S. E.; Sussmann, R. S. Diamond Relat. Mater. 2000, 9 (910), 1726-1729.

(7) Jelezko, F.; Wrachtrup, J. Phys. Status Solidi A 2006, 203 (13), $3207-3225$.

(8) Kurtsiefer, C.; Mayer, S.; Zarda, P.; Weinfurter, H. Phys. Rev. Lett. 2000, 85 (2), 290-293.

(9) Babinec, T. M.; Hausmann, B. J. M.; Khan, M.; Zhang, Y.; Maze, J. R.; Hemmer, P. R.; Loncar, M. Nat. Nanotechnol. 2010, 5 (3), 195199.

(10) Dutt, M. V. G.; Childress, L.; Jiang, L.; Togan, E.; Maze, J.; Jelezko, F.; Zibrov, A. S.; Hemmer, P. R.; Lukin, M. D. Science 2007, 316 (5829), 1312-1316.

(11) Aharonovich, I.; Greentree, A. D.; Prawer, S. Nat. Photonics 2011, 5 (7), 397-405.

(12) Greentree, A. D.; Fairchild, B. A.; Hossain, F. M.; Prawer, S. Mater. Today 2008, 11 (9), 22-31.

(13) Liao, M.; Hishita, S.; Watanabe, E.; Koizumi, S.; Koide, Y. Adv. Mater. 2010, 22 (47), 5393-5397.

(14) Zalalutdinov, M. K.; Ray, M. P.; Photiadis, D. M.; Robinson, J. T.; Baldwin, J. W.; Butler, J. E.; Feygelson, T. I.; Pate, B. B.; Houston, B. H. Nano Lett. 2011, 11 (10), 4304-4308.

(15) Freeman, D.; Madden, S.; Luther-Davies, B. Opt. Express 2005, 13 (8), 3079-3086.

(16) Roussey, M.; Bernal, M.-P.; Courjal, N.; Baida, F. I. Appl. Phys. Lett. 2005, 87 (24), 241101-3.

(17) Babinec, T. M.; Choy, J. T.; Smith, K. J. M.; Khan, M.; LonCar, M. J. Vac. Sci. Technol. B 2011, 29 (1), 010601-6.

(18) Bayn, I.; et al. New J. Phys. 2011, 13 (2), 025018.

(19) Riedrich-Moller, J.; Kipfstuhl, L.; Hepp, C.; Neu, E.; Pauly, C.; Mucklich, F.; Baur, A.; Wandt, M.; Wolff, S.; Fischer, M.; Gsell, S.; Schreck, M.; Becher, C. Nat. Nanotechnol. 2012, 7 (1), 69-74.

(20) Bayn, I.; Meyler, B.; Lahav, A.; Salzman, J.; Kalish, R.; Fairchild, B. A.; Prawer, S.; Barth, M.; Benson, O.; Wolf, T.; Siyushev, P.; Jelezko, F.; Wrachtrup, J. Diamond Relat. Mater. 2011, 20 (7), 937943.

(21) Levy, M.; Osgood, J. R. M.; Liu, R.; Cross, L. E.; Cargill, G. S., III; Kumar, A.; Bakhru, H. Appl. Phys. Lett. 1998, 73 (16), 2293-2295.

(22) Parikh, N. R.; Hunn, J. D.; McGucken, E.; Swanson, M. L.; White, C. W.; Rudder, R. A.; Malta, D. P.; Posthill, J. B.; Markunas, R. J. Appl. Phys. Lett. 1992, 61 (26), 3124-3126.

(23) Aharonovich, I.; Lee, J. C.; Magyar, A. P.; Buckley, B. B.; Yale, C. G.; Awschalom, D. D.; Hu, E. L. Adv. Mater. 2012, 24 (10), OP54OP59.

(24) Magyar, A. P.; Lee, J. C.; Limarga, A. M.; Aharonovich, I.; Rol, F.; Clarke, D. R.; Huang, M.; Hu, E. L. Appl. Phys. Lett. 2011, 99 (8), 081913.

(25) Fairchild, B. A.; Olivero, P.; Rubanov, S.; Greentree, A. D.; Waldermann, F.; Taylor, R. A.; Walmsley, I.; Smith, J. M.; Huntington, S.; Gibson, B. C.; Jamieson, D. N.; Prawer, S. Adv. Mater. 2008, 20 (24), 4793-4798.

(26) Hiscocks, M. P.; Ganesan, K.; Gibson, B. C.; Huntington, S. T.; Ladouceur, F.; Prawer, S. Opt. Express 2008, 16 (24), 19512-19519.

(27) Liao, M.; Li, C.; Hishita, S.; Koide, Y. J. Micromech. Microeng. 2010, 20 (8), 085002.

(28) Olivero, P.; Rubanov, S.; Reichart, P.; Gibson, B. C.; Huntington, S. T.; Rabeau, J.; Greentree, A. D.; Salzman, J.; Moore, D.; Jamieson, D. N.; Prawer, S. Adv. Mater. 2005, 17 (20), 2427-2430.

(29) Wang, C. F.; Hu, E. L.; Yang, J.; Butler, J. E. J. Vac. Sci. Technol. B 2007, 25 (3), 730-733.

(30) Faraon, A.; Barclay, P. E.; Santori, C.; Fu, K.-M. C.; Beausoleil, R. G. Nat. Photonics 2011, 5 (5), 301-305.

(31) Faraon, A.; Santori, C.; Huang, Z.; Acosta, V. M.; Beausoleil, R. G. Phys. Rev. Lett. 2012, 109 (3), 033604. 
(32) Hausmann, B. J. M.; Shields, B.; Quan, Q.; Maletinsky, P.; McCutcheon, M.; Choy, J. T.; Babinec, T. M.; Kubanek, A.; Yacoby, A.; Lukin, M. D.; Loncar, M. Nano Lett. 2012, 12, 1578-1582.

(33) Ovartchaiyapong, P.; Pascal, L. M. A.; Myers, B. A.; Lauria, P.; Jayich, A. C. B. Applied Physics Letters 2012, 101 (16), 163505-4.

(34) Bayn, I.; Salzman, J.; Kalish, R. arXiv:1012.5481v1, 2010.

(35) Hausmann, B. J. M.; Khan, M.; Zhang, Y.; Babinec, T. M.; Martinick, K.; McCutcheon, M.; Hemmer, P. R.; Loncar, M. Diamond Relat. Mater. 2010, 19 (5-6), 621-629.

(36) Boyd, G. D.; Coldren, L. A.; Storz, F. G. Appl. Phys. Lett. 1980, 36 (7), 583-585.

(37) Lee, J.-K.; Lee, S.-H.; Min, J.-H.; Jang, I.-Y.; Kim, C.-K.; Moon, S. H. J. Electrochem. Soc. 2009, 156 (7), D222-D225.

(38) Takahashi, S.; Suzuki, K.; Okano, M.; Imada, M.; Nakamori, T.; Ota, Y.; Ishizaki, K.; Noda, S. Nat. Mater. 2009, 8 (9), 721-725.

(39) Huang, X. M. H. Ultrahigh and microwave frequency nanomechanical systems. Ph.D. Thesis, California Institute of Technology, Pasadena, CA, 2004.

(40) Deotare, P. B.; McCutcheon, M. W.; Frank, I. W.; Khan, M.; Loncar, M. Appl. Phys. Lett. 2009, 94 (12), 121106-3.

(41) McCutcheon, M. W.; Loncar, M. Appl. Phys. Lett. 2009, 95 (3), $031102-3$.

(42) Quan, Q.; Deotare, P. B.; Loncar, M. Appl. Phys. Lett. 2010, 96 (20), 203102-3.

(43) Quan, Q; Loncar, M. Opt. Express 2011, 19 (19), 1852918542.

(44) Lee, H.; Chen, T.; Li, J.; Yang, K. Y.; Jeon, S.; Painter, O.; Vahala, K. J. Nat. Photonics 2012, 6 (6), 369-373.

(45) Wiederhecker, G. S.; Chen, L.; Gondarenko, A.; Lipson, M. Nature 2009, 462 (7273), 633-636.

(46) Wiederhecker, G. S.; Manipatruni, S.; Lee, S.; Lipson, M. Opt. Express 2011, 19 (3), 2782-2790.

(47) Eichenfield, M.; Camacho, R.; Chan, J.; Vahala, K. J.; Painter, O. Nature 2009, 459 (7246), 550-555.

(48) Deotare, P. B.; McCutcheon, M. W.; Frank, I. W.; Khan, M.; Loncar, M. Appl. Phys. Lett. 2009, 95 (3), 031102-3. 